archives-ouvertes

\title{
Inertial Estimation and Energy-Efficient Control of a Cable-suspended Load with a Team of UAVs
}

\author{
Antonio Petitti, Dario Sanalitro, Marco Tognon, A. Milella, J Cortés, A. \\ Franchi
}

\section{To cite this version:}

Antonio Petitti, Dario Sanalitro, Marco Tognon, A. Milella, J Cortés, et al.. Inertial Estimation and Energy-Efficient Control of a Cable-suspended Load with a Team of UAVs. 2020. hal-02734823

\section{HAL Id: hal-02734823 \\ https://hal.archives-ouvertes.fr/hal-02734823}

Preprint submitted on 2 Jun 2020

HAL is a multi-disciplinary open access archive for the deposit and dissemination of scientific research documents, whether they are published or not. The documents may come from teaching and research institutions in France or abroad, or from public or private research centers.
L'archive ouverte pluridisciplinaire HAL, est destinée au dépôt et à la diffusion de documents scientifiques de niveau recherche, publiés ou non, émanant des établissements d'enseignement et de recherche français ou étrangers, des laboratoires publics ou privés. 


\title{
Inertial Estimation and Energy-Efficient Control of a Cable-suspended Load with a Team of UAVs
}

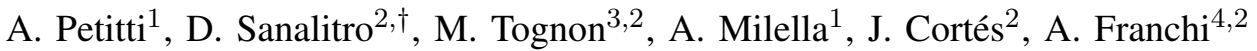

\begin{abstract}
The Fly-Crane is a multi-robot aerial manipulator system composed of three aerial vehicles towed to a platform by means of six cables. This paper presents a method to estimate the mass and the position of the center of mass of a loaded platform (i.e. the Fly-Crane platform including a transported load). The precise knowledge of these parameters allows to sensibly minimize the total effort exerted during a full-pose manipulation task The estimation is based on the measure of the forces applied by the aerial vehicles to the platform in different static configurations. We demonstrate that only two different configurations are sufficient to estimate the inertial parameters. Far-from-ideal numerical simulations show the effectiveness of the estimation method. Once the parameters are estimated, we show the enhancement of the system performances by minimizing the total exerted effort. The validity of the proposed algorithm in non-ideal conditions is presented through simulations based on the Gazebo simulator.
\end{abstract}

\section{INTRODUCTION}

In the last decades, control and estimation algorithms for the coordination of multi-robot systems have been deeply investigated [1]. Recently, the multi-robot solution became particularly popular in the field of aerial robotics. This solution increases the total payload of the system, its robustness against disturbances, as well as its manipulability, making the system suitable for construction and assembly tasks. To reduce the complexity of the system, the aerial vehicles are often connected to the load by simple cables, which allows decoupling the rotational dynamics of the vehicles to the one of the load.

Among the several designs presented in the literature, e.g., [2] and [3], the most suitable for the load full-pose control are the ones that are statically rigid [4] (or forceclosure [5]). This property allows compensating external disturbances almost instantaneously simply changing the intensity of the forces applied to the load by the cables, without changing their orientation. The minimum condition

\footnotetext{
$\dagger$ The first two authors have equally collaborated to the manuscript and can both be considered as first author.

${ }^{1}$ Institute of Intelligent Industrial Systems and Technologies for Advanced Manufacturing (STIIMA), CNR, Bari, Italy. antonio.petitti@stiima.cnr.it, annalisa.milella@ stiima.cnr.it

${ }^{2}$ LAAS-CNRS, Université de Toulouse, CNRS, Toulouse, France. dario.sanalitro@laas.fr, antonio.franchi@laas.fr

${ }^{3}$ Autonomous Systems Lab, Department of Mechanical and Process Engineering, ETH Zurich, 8092 Zürich, Switzerland, mtognon@ethz.ch

${ }^{4}$ Robotics and Mechatronics lab, Faculty of Electrical Engineering, Mathematics \& Computer Science, University of Twente, Enschede, The Netherlands a.franchi@utwente.nl

This work has been partially funded by the STIIMA "Young Ideas" grant within the project "iDEAS-3D", by the ANR, under the Project ANR-17-CE33-0007 MuRoPhen, and by the European Union's Horizon 2020 research and innovation programme under grant agreement ID: 871479 AERIAL-CORE
}

to obtain such a property is to use at least six cables. In [6], the use of one aerial vehicle per cable (at least six) is proposed. However, this solution increases the complexity and the cost of the system with respect to the case with only three pairs vehicle-cable which is the minimum requirement for full-pose control but is not statically rigid. In this work, we shall focus on a statically rigid system that uses only three aerial vehicles: the Fly-Crane [7]. The Fly-Crane, using only three vehicles connected to a platform by a pair of cables, allows to precisely control the pose of the platform while minimizing the complexity and cost of the system. A similar concept but based on bars was proposed in [8].

To perform manipulation tasks with the mentioned multirobot systems, different motion planning, and control methods have been presented. Regarding motion control, the methods range from simple flatness-based open-loop [3], full dynamic model inversion [6] and geometric techniques [9], to communication-based [1] and communication-less [10] coordination approaches. In the case of complex manipulation tasks, different motion planners have been also developed to provide desired trajectories that avoid obstacles in unknown environments [11] and ensure the feasibility of inputs and cable forces [7].

For both motion planner and controller, it turns out that an important quantity to be precisely estimated is the real position of the Center of Mass (CoM) of the load with respect to its geometric center. In fact, for model-based controllers, the knowledge of this parameter allows to better compensate for the gravity effects. For motion planners, this parameter is instead important to compute the configuration that best shares the weight of the load among the aerial vehicles. Due to the limited payload of the vehicles, it is advisable that each aerial vehicle provides nearly the same force to sustain the load, thus optimizing the energy-efficiency and flight-time autonomy.

To improve both tracking performance and the generation of optimal trajectories, our main contribution is the design of an observer that can estimate the position of the CoM of the loaded platform with respect to its geometric center. For this observer, we analyze the observability conditions necessary to estimate the CoM. We show that two different static configurations are sufficient for the estimation. This observer can run in a first initialization phase if the position of the CoM is constant, or during task-execution, if the position of the CoM changes over time (e.g., for pick \& place operations). The estimated quantity is then used in an online optimizer that, given the desired pose trajectory of the loaded platform, computes the remaining configuration 


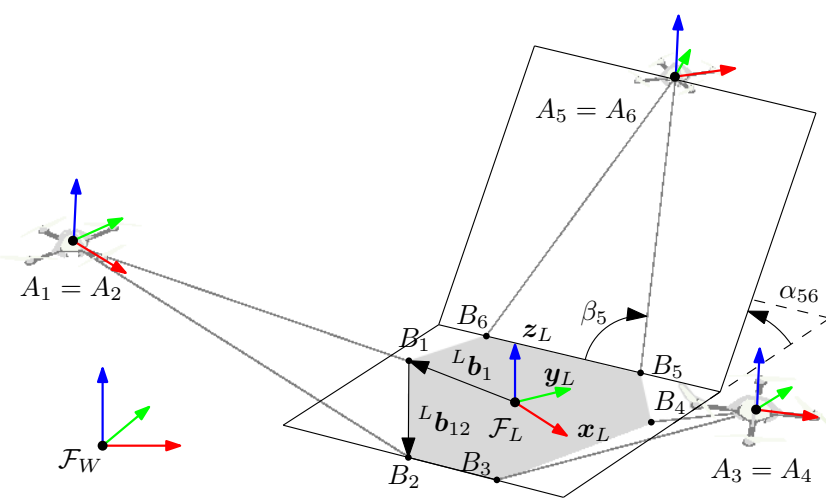

Fig. 1: Schematic representation of the Fly-Crane and its main variables.

variables (the angles of the cables) such that the weight of the loaded platform is equally balanced among the robots. Finally, the computed desired configuration is tracked by a robust controller, presented in [12], capable of dealing with external disturbances and non-idealities arising from modeling errors at the dynamic, and kinematic levels.

The proposed framework, and in particular the estimation method for the position of the loaded platform CoM, has been tested with numerical simulation in non-ideal conditions. Through these simulations, we shall highlight the positive impact of improving the parameters estimation.

The manuscript is organized as follows. Section II describes the Fly-Crane system and its modeling, while in Sec. III the proposed control and optimization strategy is presented. Section IV introduces the proposed estimation strategy together with the observability analysis. The simulation results in non-ideal conditions are shown in Sec. V. Finally, discussions and future works are presented in Sec. VI.

\section{SYSTEM MODELING}

The Fly-Crane system consists of three aerial vehicles attached to a platform by six cable. The platform is equipped with an additional load. Each aerial vehicle is tied to the loaded platform by two cables. The system is schematically depicted in Fig. 1. Connections between cable-platform and cable-aerial vehicle are done such that no rotational constraints are present.

In order to describe the system, let us define an inertial frame $\mathcal{F}_{W}=\left\{O_{W}, \boldsymbol{x}_{W}, \boldsymbol{y}_{W}, \boldsymbol{z}_{W}\right\}$, where $O_{W}$ is its origin and $\left\{\boldsymbol{x}_{W}, \boldsymbol{y}_{W}, \boldsymbol{z}_{W}\right\}$ are its unit axes. We also define the frame $\mathcal{F}_{L}=\left\{O_{L}, \boldsymbol{x}_{L}, \boldsymbol{y}_{L}, \boldsymbol{z}_{L}\right\}$ rigidly attached to the platform. In particular, $O_{L}$ is the origin of $\mathcal{F}_{L}$ and $\left\{\boldsymbol{x}_{L}, \boldsymbol{y}_{L}, \boldsymbol{z}_{L}\right\}$ are its unit axes. $\mathcal{F}_{L}$ is placed such that $O_{L}$ is in the geometric center of the platform and $z_{L}$ is perpendicular to the platform plane. The vector ${ }^{W} \boldsymbol{p}_{L} \in \mathbb{R}^{3}$ describes the position of $O_{L}$ with respect to $\mathcal{F}_{W}$ and ${ }^{W} \boldsymbol{R}_{L} \in S O(3)$ describes the orientation of $\mathcal{F}_{L}$ with respect to $\mathcal{F}_{W}{ }^{1}$. It is worth noticing that, if the (loaded) platform's mass is uniformly distributed, the geometric center $O_{L}$ will coincide with the CoM of the

\footnotetext{
${ }^{1}$ The left superscript indicates the reference frame. From now on, $\mathcal{F}_{W}$ is considered as reference frame when the superscript is omitted.
}

platform. However, this is rarely the case, especially when the platform is used for the manipulation of objects.

Let us denote with $C$ the CoM of the platform and with $p_{C}$ the position of $C$ with respect to $\mathcal{F}_{W}$ or, equivalently, with ${ }^{L} \boldsymbol{p}_{C}$ the position of $C$ with respect to $\mathcal{F}_{L}$.

As already introduced, six cables are rigidly attached to the platform, pulling it in such a way to control its position and orientation. We assume that the cables have negligible mass and inertia with respect to the other bodies of the system. The $i$-th cable, with $i=1, \ldots, 6$, is attached at one end to the platform at the point $B_{i}$ and at the other end to an aerial vehicle at the point $A_{i}$. The point $B_{i}$ and $A_{i}$ are described by the vectors $\boldsymbol{b}_{i} \in \mathbb{R}^{3}$ and $\boldsymbol{p}_{R i} \in \mathbb{R}^{3}$ with respect to $\mathcal{F}_{W}$, respectively, or equivalently by the vectors ${ }^{L} \boldsymbol{b}_{i} \in \mathbb{R}^{3}$ and ${ }^{L} \boldsymbol{p}_{R i} \in \mathbb{R}^{3}$ with respect to $\mathcal{F}_{L}$. Assuming deformations due to elasticity negligible in the operative conditions, the $i$-th cable is characterized by a constant length $l_{i} \in \mathbb{R}_{>0}$.

We denote with $f_{L i} \in \mathbb{R}_{>0}$ the intensity of the internal force along the $i$-th cable. Notice that if $f_{L i}>0$ the cable is taut, while is slack if $f_{L i}=0$. Therefore, the motion of the system has to be planned in a way to preserve the tautness of each cable, i.e., such that $f_{L i}>0$ for all $i=1, \ldots, 6$.

We consider the aerial vehicles as thrust generation units where $\boldsymbol{f}_{R i j} \in \mathbb{R}^{3}$ is the $3 \mathrm{D}$ controllable total thrust vector of each vehicle.

Assuming the cables always taut, we have that each pair of cables lays on a plane, as depicted in Fig. 1. The configuration of the pair of cables $(i, j)$ is then given by the angles $\alpha_{i j} \in \mathbb{R}$ between the plane formed by the cables and the one composed by the axis $\left\{\boldsymbol{x}_{L}, \boldsymbol{y}_{L}\right\}$. Given the previous description of the system and the relative constraints, the platform configuration can be entirely described by $\left(\boldsymbol{p}_{L}, \boldsymbol{R}_{L}, \boldsymbol{\alpha}\right) \in$ $\mathcal{C}=S E(3) \times \mathbb{R}^{3}$, where $\boldsymbol{\alpha}=\left[\begin{array}{lll}\alpha_{12} & \alpha_{34} & \alpha_{56}\end{array}\right]^{\top} \in \mathbb{R}^{3}$. Let us indicate with $\boldsymbol{q}=\left[\boldsymbol{p}_{L}^{\top} \boldsymbol{\eta}^{\top} \boldsymbol{\alpha}^{\top}\right]^{\top}$ the generalized coordinates of the system, where $\boldsymbol{\eta}=\left[\begin{array}{lll}\phi & \theta\end{array}\right]^{\top}$ is a Euler angle parametrization of $\boldsymbol{R}_{L}$. The only undetermined variable, i.e. the position of the aerial vehicles $\boldsymbol{p}_{R i}$, can be easily computed by direct kinematics from $\boldsymbol{q}$ :

$$
\boldsymbol{p}_{R i}(\boldsymbol{q})=\boldsymbol{p}_{L}+\boldsymbol{R}_{L}{ }^{L} \boldsymbol{p}_{R i}\left(\alpha_{i j}\right),
$$

where ${ }^{L} \boldsymbol{p}_{R i}={ }^{L} \boldsymbol{b}_{i}+l_{i} \boldsymbol{R}_{{ }^{L}} \boldsymbol{b}_{i j}\left(\alpha_{i j}\right) \boldsymbol{R}_{\boldsymbol{z}_{L}}\left(\beta_{i}\right) \frac{{ }^{L} \boldsymbol{b}_{i j}}{\left\|\boldsymbol{b}_{i j}\right\|},{ }^{L} \boldsymbol{b}_{i j}$ is the vector $\overrightarrow{B_{i} B_{j}}$ expressed in $\mathcal{F}_{L}$ and $\beta_{i} \in \mathbb{T}$ is the angle between $\overrightarrow{B_{i} B_{j}}$ and $\overrightarrow{B_{i} A_{i}}$. The rotation matrices $\boldsymbol{R}_{L}{ }_{\boldsymbol{b}_{i j}}\left(\alpha_{i j}\right)$ and $\boldsymbol{R}_{\boldsymbol{z}_{L}}\left(\beta_{i}\right)$ represent, respectively, the rotation of $\alpha_{i j}$ about axis ${ }^{L} \boldsymbol{b}_{i j}$ and the rotation of $\beta_{i}$ about axis $\boldsymbol{z}_{L}$. Let $\boldsymbol{v}=$ $\left[\begin{array}{lll}\dot{\boldsymbol{p}}_{R 1}^{\top} & \dot{\boldsymbol{p}}_{R 2}^{\top} & \dot{\boldsymbol{p}}_{R 3}^{\top}\end{array}\right]^{\top}$ be the velocities of the aerial vehicles, then, the following holds:

$$
\boldsymbol{v}=\boldsymbol{J}(\boldsymbol{q}) \dot{\boldsymbol{q}},
$$

where $\mathbf{J} \in \mathbb{R}^{9 \times 9}$ is the Jacobian matrix defined as

$$
\mathbf{J}=\left[\begin{array}{lll}
\boldsymbol{I}_{3} & \mathbf{J}_{12} & \mathbf{R}_{12} \\
\boldsymbol{I}_{3} & \mathbf{J}_{34} & \mathbf{R}_{34} \\
\boldsymbol{I}_{3} & \mathbf{J}_{56} & \mathbf{R}_{56}
\end{array}\right],
$$




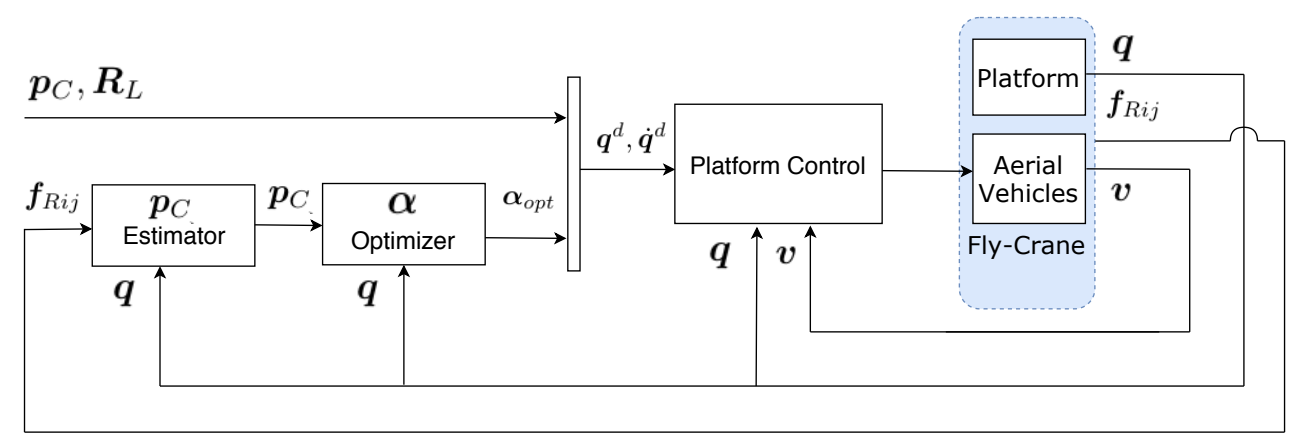

Fig. 2: The control architecture of the Fly-Crane with highlighted the CoM estimator, the $\alpha$ optimizer, the configuration controller of the platform and the velocity controllers of the three aerial vehicles.

where $\boldsymbol{I}_{n} \in \mathbb{R}^{n \times n}$ is the identity matrix of dimension $n$,

$$
\begin{gathered}
\mathbf{J}_{i j}=-\left[\boldsymbol{R}_{L}\left({ }^{L} \boldsymbol{b}_{i}+l_{i} \boldsymbol{R}_{L} \boldsymbol{b}_{i j}\left(\alpha_{i j}\right) \boldsymbol{R}_{\boldsymbol{z}_{L}}\left(\beta_{i}\right) \frac{{ }^{L} \boldsymbol{b}_{i j}}{\left\|^{L} \boldsymbol{b}_{i j}\right\|}\right)\right]_{\times}, \\
\mathbf{R}_{i j}=-l_{i} \boldsymbol{R}_{L} \boldsymbol{R}_{{ }^{L} \boldsymbol{b}_{i j}}\left(\alpha_{i j}\right)\left[\frac{{ }^{L} \boldsymbol{b}_{i j}}{\left\|^{L} \boldsymbol{b}_{i j}\right\|}\right]_{\times} \boldsymbol{R}_{\boldsymbol{z}_{L}}\left(\beta_{i}\right) \frac{{ }^{L} \boldsymbol{b}_{i j}}{\left\|^{L} \boldsymbol{b}_{i j}\right\|} .
\end{gathered}
$$

For a given vector $\boldsymbol{\nu}=\left[\begin{array}{lll}\nu_{x} & \nu_{y} & \nu_{z}\end{array}\right]^{\top}$, the notation $[\boldsymbol{\nu}]_{\times}$ indicates the skew-symmetric matrix.

Furthermore, thanks to the Jacobian matrix $\mathbf{J}$, following [13], we can write the dynamics of the whole system, including platform and aerial vehicles as

$$
\boldsymbol{M} \ddot{\boldsymbol{q}}+\boldsymbol{c}+\boldsymbol{n}=\mathbf{J}^{\top}\left[\begin{array}{l}
\boldsymbol{f}_{R 12} \\
\boldsymbol{f}_{R 34} \\
\boldsymbol{f}_{R 56}
\end{array}\right],
$$

where $M \in \mathbb{R}^{9 \times 9}$ is the generalized inertia matrix of the system, $c \in \mathbb{R}^{9}$ is the vector of Coriolis and centrifugal terms and $\boldsymbol{n} \in \mathbb{R}^{9}$ accounts for gravitational effects. Analyzing (4), we remark that a non-zero displacement of $C$ from $O_{L}$ (if ${ }^{L} \boldsymbol{p}_{C} \neq \mathbf{0}$ ) induces an additional torque component $\boldsymbol{R}_{L}{ }^{L} \boldsymbol{p}_{C} \times m_{L} \boldsymbol{g}$, where $m_{L}$ is the mass of the loaded platform, and $\boldsymbol{g}$ is the gravity vector. This is more evident if we make explicit $\boldsymbol{n}$

$$
\boldsymbol{n}\left({ }^{L} \boldsymbol{p}_{C}, \boldsymbol{q}\right)=m_{L}\left[\begin{array}{c}
\boldsymbol{g} \\
\boldsymbol{R}_{L}{ }^{L} \boldsymbol{p}_{C} \times \boldsymbol{g} \\
\mathbf{0}
\end{array}\right]+m_{Q} \mathbf{J}^{T}\left[\begin{array}{l}
\boldsymbol{g} \\
\boldsymbol{g} \\
\boldsymbol{g}
\end{array}\right],
$$

where $m_{Q}$ is the mass of each robot (which is considered the same for ease of notation).

\section{CONTROL AND OPTIMIZATION STRATEGY}

The Fly-Crane can be seen as a redundant manipulator, since the desired pose of the platform (i.e. the end-effector) can be achieved with an infinity of configurations. The angles $\boldsymbol{\alpha}$ represent the 3-DoF redundancy of the system. Here, we propose a method to locally optimize the desired value of these angles, $\boldsymbol{\alpha}^{d}$, in order to balance the loaded platform's weight among all vehicles. In the following, we call this method $\boldsymbol{\alpha}^{d}$ optimizer. The estimated center of mass and the orientation of the loaded platform play a fundamental role to compute the optimal $\boldsymbol{\alpha}^{d}$. Therefore they represent the inputs of the $\boldsymbol{\alpha}^{d}$ optimizer as depicted in Fig. 2. The values of $\boldsymbol{\alpha}^{d}$ are computed by solving the following minimization problem:

$$
\begin{gathered}
\boldsymbol{\alpha}_{o p t}=\arg \min _{\boldsymbol{\alpha}} \mu\left({ }^{L} \boldsymbol{p}_{C}, \boldsymbol{q}\right), \\
\text { s.t. } 0 \leq \alpha_{i j}<\frac{\pi}{2}, \quad \text { for }(i, j) \in\{(1,2),(4,3),(5,6)\},
\end{gathered}
$$

where $\mu\left({ }^{L} \boldsymbol{p}_{C}, \boldsymbol{q}\right)=\left|\left\|\boldsymbol{f}_{R 12}\right\|-\left\|\boldsymbol{f}_{R 34}\right\|\right|+\mid\left\|\boldsymbol{f}_{R 34}\right\|-$ $\left\|\boldsymbol{f}_{R 56}\right\||+|\left\|\boldsymbol{f}_{R 56}\right\|-\left\|\boldsymbol{f}_{R 12}\right\| \mid$. It is worth noting that this function reaches its minimum when the norms of the forces are the same, i.e., each aerial vehicle is equally contributing to the transportation effort.

In this work, we apply a simple gradient descent iterative algorithm to solve the optimization problem, but more sophisticated techniques could be used. More precisely, at each time instant, we vary the value of $\boldsymbol{\alpha}$ of a quantity proportional to the approximate anti-gradient of $\mu\left({ }^{L} \boldsymbol{p}_{C}, \boldsymbol{q}\right)$.

At this point, the available desired trajectories $\boldsymbol{q}^{d}=$ $\left[\begin{array}{lll}\boldsymbol{p}_{L}^{d} & \boldsymbol{\eta}^{d} & \boldsymbol{\alpha}_{\text {opt }}^{d}\end{array}\right]$ and $\dot{\boldsymbol{q}}^{d}=\left[\begin{array}{lll}\dot{\boldsymbol{p}}_{L}^{d} & \dot{\boldsymbol{\eta}}^{d} & \dot{\boldsymbol{\alpha}}_{\text {opt }}^{d}\end{array}\right]$ are fed to the controller which generates the commanded acceleration for each vehicle $\boldsymbol{u}=\left[\begin{array}{lll}\boldsymbol{u}_{1}^{\top} & \boldsymbol{u}_{2}^{\top} & \boldsymbol{u}_{3}^{\top}\end{array}\right]^{\top}$. Such commanded accelerations are provided to the low-level actuation units of the aerial vehicles. Each low-level controller takes care of the orientation and total thrust of the vehicles by controlling the rotor speeds aiming at implementing the desired linear acceleration. In the following we detail the equations used in each level of the control framework represented in Fig. 2. Given the desired configuration $\boldsymbol{q}^{d}$, the corresponding generalized velocities $\dot{\boldsymbol{q}}^{d}$, and the measured configuration $\boldsymbol{q}$, the commanded accelerations are computed simply as

$$
\boldsymbol{u}=\boldsymbol{K}_{R}\left(\boldsymbol{J}(\boldsymbol{q})\left(\boldsymbol{K}_{\boldsymbol{q}} \boldsymbol{e}_{\boldsymbol{q}}+\dot{\boldsymbol{q}}^{d}\right)-\boldsymbol{v}\right)
$$

where $\boldsymbol{K}_{\boldsymbol{q}}=k_{\boldsymbol{q}} \boldsymbol{I}_{9} \in \mathbb{R}_{>0}^{9 \times 9}$ and $\boldsymbol{K}_{R}=k_{R} \boldsymbol{I}_{9} \in \mathbb{R}_{>0}^{9 \times 9}$ are positive definite matrices representing control gains, and $\boldsymbol{e}_{\boldsymbol{q}}=\boldsymbol{q}^{d}-\boldsymbol{q}$.

The convergence and stability of the implemented controller have been proven in [12]. 


\section{ESTIMATION OF THE INERTIAL PARAMETERS}

In this section, the estimation of the inertial parameters needed to optimize the energy exerted by the system taking advantage of the Fly-Crane redundancy. First, the necessary conditions for the estimation are demonstrated. The proposed estimation strategy is described afterward.

Let us model the loaded platform of the Fly-Crane as a rigid body on which six forces $\mathbf{f}_{i}=f_{L i} \boldsymbol{\kappa}_{i}$ are acting in $B_{i}$, respectively, with $i=1, \ldots, 6$, and $\boldsymbol{\kappa}_{i}=\frac{\boldsymbol{p}_{R i}-\boldsymbol{b}_{i}}{\left\|\boldsymbol{p}_{R i}-\boldsymbol{b}_{i}\right\|} \in \mathrm{SO}(2)$ being the unit vector representing the attitude of the cable. For ease of exposition, we introduce a new reference frame $\mathcal{F}_{C}=\left\{O_{C}, \boldsymbol{x}_{C}, \boldsymbol{y}_{C}, \boldsymbol{z}_{C}\right\}$, where its origin $O_{C}$ is in $C$ and $\left\{\boldsymbol{x}_{C}, \boldsymbol{y}_{C}, \boldsymbol{z}_{C}\right\}$ are its unit axes. $\mathcal{F}_{C}$ is oriented as $\mathcal{F}_{L}$. Thus, the vector $p_{C}$ also describes the position of $O_{C}$ with respect to $\mathcal{F}_{W}$ and $\boldsymbol{R}_{C} \in S O(3)$ describes the orientation of $\mathcal{F}_{C}$ with respect to $\mathcal{F}_{W}$, resulting that $\boldsymbol{R}_{C}=\boldsymbol{R}_{L}$. Therefore, the dynamical model of the loaded platform, with reference to $\mathcal{F}_{C}$, is the one of a rigid body subject to the forces $\mathbf{f}_{i}$, $i=1, \ldots, 6$, i.e.,

$$
\left\{\begin{array}{rl}
m_{L} \dot{\mathbf{v}}_{C} & =\sum_{i=1}^{6} \mathbf{f}_{i}+m_{L} \mathbf{g} \\
\mathbf{I} \dot{\boldsymbol{\omega}}+\boldsymbol{\omega} \times \mathbf{I} \boldsymbol{\omega} & =\sum_{i=1}^{6}\left[\left(\boldsymbol{b}_{i}-\mathbf{p}_{C}\right) \times \mathbf{f}_{i}\right]
\end{array},\right.
$$

where $\dot{\mathbf{v}}_{C}$ is the velocity of $C, \boldsymbol{\omega}$ is the angular velocity of the loaded platform, and $\mathbf{I}$ is the inertia tensor of the loaded platform. It is worth noting that in (8) there are no torques due to gravity. Seen from $\mathcal{F}_{C}$, gravity acts in the origin $C$. The forces $\mathbf{f}_{i}, i=1, \ldots, 6$ are obtained from the robot thrusts inverting (4). Moreover, we assume that the wrench-feasibility constraints and the thrust constraints are satisfied [7]. The equations of the statics, in $\mathcal{F}_{C}$, are the following:

$$
\left\{\begin{array}{rl}
\sum_{i=1}^{6}{ }^{C} \mathbf{f}_{i} & =-m_{L} \boldsymbol{R}_{C}^{T} \mathbf{g} \\
\sum_{i=1}^{6}\left({ }^{C} \boldsymbol{b}_{i} \times{ }^{C} \mathbf{f}_{i}\right) & =\mathbf{0}
\end{array} .\right.
$$

Let us note that ${ }^{C} \boldsymbol{b}_{i}={ }^{L} \boldsymbol{b}_{i}-{ }^{L} \boldsymbol{p}_{C}$ and ${ }^{C} \mathbf{f}_{i}={ }^{L} \mathbf{f}_{i}$. Thus, (9) can be suitably written as

$$
\left\{\begin{array}{rl}
\sum_{i=1}^{6}{ }^{{ }^{L}} \mathbf{f}_{i} & =-m_{L} \boldsymbol{R}_{L}^{T} \mathbf{g} \\
\sum_{i=1}^{6}\left[\left({ }^{L} \boldsymbol{b}_{i}-{ }^{L} \boldsymbol{p}_{C}\right) \times{ }^{L} \mathbf{f}_{i}\right] & =\mathbf{0}
\end{array} .\right.
$$

Then, we can write (10) as a linear system $\mathbf{A x}=\mathbf{b}$, where $\mathbf{x}=\left[\begin{array}{llll}m_{L}^{-1} & { }^{L} p_{C, x} & { }^{L} p_{C, y} & { }^{L} p_{C, z}\end{array}\right]^{T} \in \mathbb{R}^{4}$, $\mathbf{b}=\left[\begin{array}{ll}-\left(\boldsymbol{R}_{L}^{T} \mathbf{g}\right)^{T} & -\sum_{i=1}^{6}\left({ }^{L} \boldsymbol{b}_{i} \times{ }^{L} \mathbf{f}_{i}\right)^{T}\end{array}\right]^{T} \in \mathbb{R}^{6}$, and

$$
\mathbf{A}=\left[\begin{array}{cc}
\sum_{i=1}^{6}{ }^{L} \mathbf{f}_{i} & \mathbf{0} \\
\mathbf{0} & {\left[\sum_{i=1}^{6}{ }^{L} \mathbf{f}_{i}\right]_{\times}}
\end{array}\right] \in \mathbb{R}^{6 \times 4} .
$$

It is worth noting that $\mathbf{A}$ and $\mathbf{b}$ strongly depend on the configuration of the system and, in particular, on the orientation of the loaded platform through $\boldsymbol{R}_{L}$. Given the dimension of the unknown vector $\mathbf{x}$, it may seem that a single configuration is sufficient to estimate the parameters. However, $\mathbf{A}$ is not full rank. Let us note that $\mathbf{A}$ can be seen as an upper triangular block matrix, thus (Theorem 3.10 in [14])

$$
\operatorname{rank}(\mathbf{A})=\operatorname{rank}\left(\sum_{i=1}^{6}{ }^{L} \mathbf{f}_{i}\right)+\operatorname{rank}\left(\left[\sum_{i=1}^{6}{ }^{L} \mathbf{f}_{i}\right]_{\times}\right)
$$

and it follows that $\operatorname{rank}(\mathbf{A})=3$. Therefore, in order to estimate $\mathbf{x}$, we need at least two different static configurations, namely, $\overline{\boldsymbol{q}}_{1}$ and $\overline{\boldsymbol{q}}_{2}$ leading to, respectively, the following two pairs $\left(\mathbf{A}_{1}, \mathbf{b}_{1}\right)$ and $\left(\mathbf{A}_{2}, \mathbf{b}_{2}\right)$. However, $\overline{\boldsymbol{q}}_{1}$ and $\overline{\boldsymbol{q}}_{2}$ must be chosen in such a way to guarantee that $\widetilde{\mathbf{A}}=\left[\begin{array}{ll}\mathbf{A}_{1}^{T} & \mathbf{A}_{2}^{T}\end{array}\right]^{T} \in \mathbb{R}^{12 \times 4}$ is full rank. To this aim, we use the Gramian matrix, defined as $\mathbf{G}=\widetilde{\mathbf{A}}^{T} \widetilde{\mathbf{A}}$, to analyze linear independence of vectors in $\widetilde{\mathbf{A}}$, that is to say, a set of vectors are linearly independent if and only if the Gramian matrix is full rank [15]. Thus, the eigenvalues of $\mathbf{G}$ give us information about $\widetilde{\mathbf{A}}$. Specifically, if the smallest eigenvalue $\lambda_{\min }$ of $\mathbf{G}$ is non zero, then $\mathbf{x}$ can be estimated. Indicated with $\mathbf{f}_{i}(\overline{\boldsymbol{q}})$ the resulting force acting on the loaded platform given the configuration $\overline{\boldsymbol{q}}$, we state the following proposition.

Proposition 1. Given two configurations $\overline{\boldsymbol{q}}_{1}$ and $\overline{\boldsymbol{q}}_{2}$, the unknown vector $\mathbf{x}=\left[\begin{array}{llll}m_{L}^{-1} & { }^{L} p_{C, x} & { }^{L} p_{C, y} & { }^{L} p_{C, z}\end{array}\right]^{T}$ can be estimated if and only if $\sum_{i=1}^{6}{ }^{L} \mathbf{f}_{i}\left(\overline{\boldsymbol{q}}_{1}\right) \neq \sum_{i=1}^{6}{ }^{{ }^{L}} \mathbf{f}_{i}\left(\overline{\boldsymbol{q}}_{2}\right)$.

Proof. Let us analyze the Gramian matrix $\mathbf{G}$ defined as $\mathbf{G}=$ $\widetilde{\mathbf{A}}^{T} \tilde{\mathbf{A}}$. We note that $\mathbf{A}_{1}$ and $\mathbf{A}_{2}$ have the same structure

$$
\mathbf{A}_{1}=\left[\begin{array}{cc}
\boldsymbol{\rho} & \mathbf{0} \\
\mathbf{0} & {[\boldsymbol{\rho}]_{\times}}
\end{array}\right], \mathbf{A}_{2}=\left[\begin{array}{cc}
\boldsymbol{\sigma} & \mathbf{0} \\
\mathbf{0} & {[\boldsymbol{\sigma}]_{\times}}
\end{array}\right],
$$

where $\boldsymbol{\rho}=\sum_{i=1}^{6}{ }^{L} \mathbf{f}_{i}\left(\overline{\boldsymbol{q}}_{1}\right), \boldsymbol{\sigma}=\sum_{i=1}^{6}{ }^{L_{i}} \mathbf{f}_{i}\left(\overline{\boldsymbol{q}}_{2}\right)$. Thus, G presents the following structure

$$
\mathbf{G}=\left[\begin{array}{cc}
\boldsymbol{\rho}^{T} \boldsymbol{\rho}+\boldsymbol{\sigma}^{T} \boldsymbol{\sigma} & \mathbf{0} \\
\mathbf{0} & \left.[\boldsymbol{\rho}]_{\times}^{T}[\boldsymbol{\rho}]_{\times}+[\boldsymbol{\sigma}]_{\times}^{T}[\boldsymbol{\sigma}]_{\times}\right] .
\end{array}\right.
$$

The eigenvalues of $\mathbf{G}$, indicated with $\operatorname{eig}(\mathbf{G})$, are

$$
\operatorname{eig}(\mathbf{G})=\left\{\gamma, \gamma, \frac{\gamma}{2} \pm \frac{1}{2} \xi^{\frac{1}{2}}\right\}
$$

where $\gamma=\boldsymbol{\rho}^{T} \boldsymbol{\rho}+\boldsymbol{\sigma}^{T} \boldsymbol{\sigma}$ and $\xi=\left(\boldsymbol{\rho}^{T} \boldsymbol{\rho}+\boldsymbol{\sigma}^{T} \boldsymbol{\sigma}\right)^{2}+$ $4\left(\boldsymbol{\rho}^{T} \boldsymbol{\sigma}\right)^{2}-4 \boldsymbol{\rho}^{T} \boldsymbol{\rho} \boldsymbol{\sigma}^{T} \boldsymbol{\sigma}$ (see the Appendix for a detailed analysis of the eigenvalues of $\mathbf{G}$ ). Hence, $\lambda_{\min }=0$ iff $\xi=\gamma^{2}$ :

$$
\begin{aligned}
\xi & =\gamma^{2} & \Rightarrow \\
\gamma^{2}+4\left(\boldsymbol{\rho}^{T} \boldsymbol{\sigma}\right)^{2}-4 \boldsymbol{\rho}^{T} \boldsymbol{\rho} \boldsymbol{\sigma}^{T} \boldsymbol{\sigma} & =\gamma^{2} & \Rightarrow \\
\left(\boldsymbol{\rho}^{T} \boldsymbol{\sigma}\right)^{2} & =\boldsymbol{\rho}^{T} \boldsymbol{\rho} \boldsymbol{\sigma}^{T} \boldsymbol{\sigma} & \Rightarrow \\
\boldsymbol{\rho}^{T}\left(\boldsymbol{\sigma} \boldsymbol{\rho}^{T}\right) \boldsymbol{\sigma} & =\boldsymbol{\rho}^{T}\left(\boldsymbol{\rho} \boldsymbol{\sigma}^{T}\right) \boldsymbol{\sigma} & \Rightarrow \\
\boldsymbol{\sigma} \boldsymbol{\rho}^{T} & =\boldsymbol{\rho} \boldsymbol{\sigma}^{T} &
\end{aligned},
$$

implying that $\mathbf{G}$ is singular if and only if $\rho=\sigma$. Then, the proposition is proved.

To give a physical interpretation to Proposition 1 , observe that we can rewrite the condition $\sum_{i=1}^{6}{ }^{L} \mathbf{f}_{i}\left(\overline{\boldsymbol{q}}_{1}\right) \neq$ 
$\sum_{i=1}^{6}{ }^{L} \mathbf{f}_{i}\left(\overline{\boldsymbol{q}}_{2}\right)$ as $\boldsymbol{R}_{L 1}^{\top} \mathbf{g} \neq \boldsymbol{R}_{L 2}^{\top} \mathbf{g}$, where $\boldsymbol{R}_{L 1}, \boldsymbol{R}_{L 2}$ indicate the rotation matrices associated to the configuration $\overline{\boldsymbol{q}}_{1}, \overline{\boldsymbol{q}}_{2}$ respectively. Thus, $\overline{\boldsymbol{R}}_{L 1}$ and $\overline{\boldsymbol{R}}_{L 2}$ cannot represent a rotation around $z_{W}$ at the same time. This clarifies as platform rotations around a vector parallel to the $\mathrm{g}$ vector do not contribute in the estimation process. Indeed, as a result of such rotations, the load balancing does not change.

Guided by this result, we propose the following estimation algorithm composed by two phases: the initialization phase and the online estimation phase reported in Algorithm 1 and Algorithm 2, respectively. The introduction of a preliminary initialization phase is motivated by the possibility that the desired trajectory to follow does not provide an opportune variation of the orientation of the loaded platform. Thus, resulting in no informative data for the estimation procedure. For this reason, we introduce an initialization phase in order to have at least an initial estimation of ${ }^{L} \boldsymbol{p}_{C}$. On the other hand, the online estimation phase aims to improve the estimation by filtering out possible measurement noise.
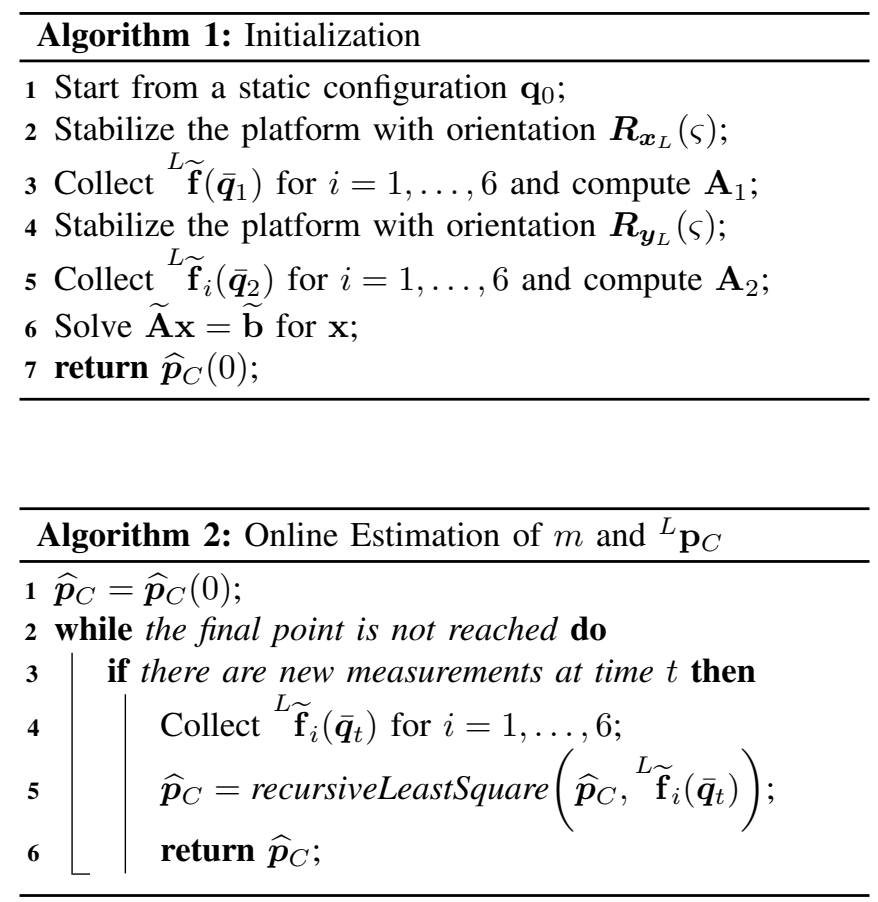

The initialization phase assumes to start from a generic configuration $\mathbf{q}^{\star}=\left[\begin{array}{llllll}\mathbf{p}_{L \star}^{T} & 0 & 0 & 0 & \alpha_{\star} & \alpha_{\star} \\ \alpha_{\star}\end{array}\right]^{T}$. Then, two different orientations $\boldsymbol{R}_{\boldsymbol{x}_{L}}(\varsigma)$ and $\boldsymbol{R}_{\boldsymbol{y}_{L}}(\varsigma)$ of the platform are imposed through the controller where $\boldsymbol{R}_{\boldsymbol{x}_{L}}(\varsigma)$ is the rotation of angle $\varsigma>0$ about axis $\boldsymbol{x}_{L}$ and $\boldsymbol{R}_{\boldsymbol{y}_{L}}(\varsigma)$ is similarly defined. Then, the measurements acquired in these two different configurations are used to set the initial value $\widehat{\boldsymbol{p}}_{C}(0)$ of the estimate $\widehat{\boldsymbol{p}}_{C}$ of $\boldsymbol{p}_{C}$. It is worth to notice that during the initialization phase, we do not perform the optimization procedure. On the other hand, the online phase is employed, starting from $\widehat{\boldsymbol{p}}_{C}(0)$, to update of the estimate $\widehat{p}_{C}$. Anytime new measurements are available, the estimate $\widehat{\boldsymbol{p}}_{C}$ is updated by means of a standard Recursive Least Square procedure [16].

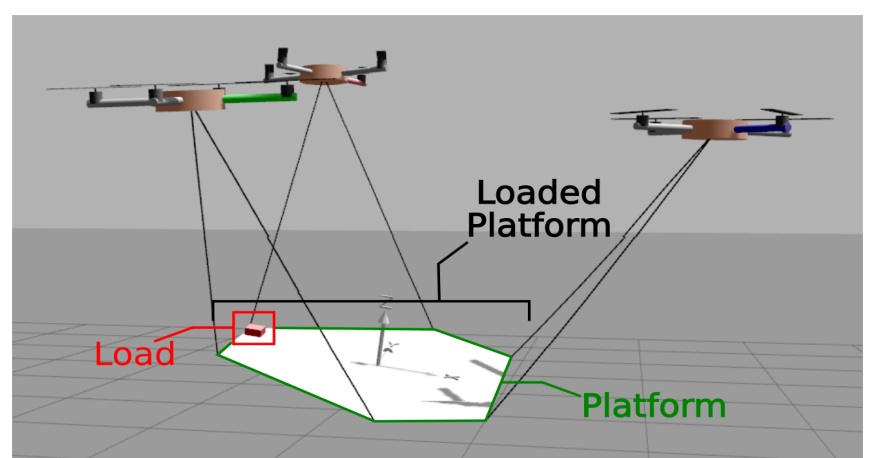

Fig. 3: Screenshot of the Fly-Crane system simulated in Gazebo.

\section{NUMERICAL AND SIMULATION RESULTS}

In this section, we describe the validation of the estimation algorithm, introduced in Sec. IV, and of the optimization strategy, given in Sec. III, by means of numerical simulations. To this aim, first, we simulate the estimation of the loaded platform mass and its center of mass. Then, we use this estimation to compute the value of $\boldsymbol{\alpha}$ that locally optimizes the force distribution among the robots.

\section{A. Simulation Scenario}

We consider a simulation scenario suitable for the execution of trajectory tracking tasks. It is implemented using Gazebo, modeling the cables as a set of serially-connected links, such that non-ideal effects as cable deformations, cable vibrations and sagging, and noisy measurements are taken into account. An image of the simulation scenario is shown in Fig. 3. We perform our tests with software in the loop: the low-level controller of the aerial vehicles (based on the Tele Ky $b^{2}$ framework) is the one running on board in our real platforms whose hardware interface is emulated as well. The high-level controller is implemented in MATLAB/Simulink. The low-level controller runs at a frequency of $1[\mathrm{kHz}]$, while the high-level controller runs at a frequency of $5[\mathrm{~Hz}]$. The system has been simulated using the following parameters:

- the length of each cable is $1[\mathrm{~m}]$;

- the weight of each vehicle has been set to $1.03[\mathrm{~kg}]$;

- the mass of the platform is $0.2[\mathrm{~kg}]$.

Each cable is attached to the platform anchor points located in ${ }^{L} \boldsymbol{b}_{1}=\left[\begin{array}{lll}-0.433 & 0.15 & 0\end{array}\right][\mathrm{m}],{ }^{L} \boldsymbol{b}_{2}=\left[\begin{array}{lll}-0.433 & -0.15 & 0\end{array}\right][\mathrm{m}]$, ${ }^{L} \boldsymbol{b}_{3}=\left[\begin{array}{lll}0.0866 & -0.450\end{array}\right][\mathrm{m}],{ }^{L} \boldsymbol{b}_{4}=\left[\begin{array}{lll}0.3464 & -0.3 & 0\end{array}\right][\mathrm{m}]$, ${ }^{L} \boldsymbol{b}_{5}=\left[\begin{array}{llll}0.3464 & 0.3 & 0\end{array}\right][\mathrm{m}],{ }^{L} \boldsymbol{b}_{6}=\left[\begin{array}{lll}0.0866 & 0.45 & 0\end{array}\right][\mathrm{m}]$. Then, we assume a load with mass $0.2[\mathrm{Kg}]$ located in ${ }^{L} \mathbf{p}_{O}=$ $\left[\begin{array}{lll}-0.4 & 0.1 & 0\end{array}\right][\mathrm{m}]$, resulting in an increase of $100 \%$ in the loaded platform total mass. The presence of the load move the center of mass to $\mathbf{p}_{C}=\left[\begin{array}{lll}-0.2 & 0.05 & 0\end{array}\right][\mathrm{m}]$.

\section{B. Validation of the Estimation Algorithm}

Before starting with the trajectory tracking task, we run the estimation procedure in order to identify the value of the mass and the position of the CoM of the loaded platform. First, we run the initialization phase of the estimation

\footnotetext{
${ }^{2}$ https://git.openrobots.org/projects/telekyb3
} 


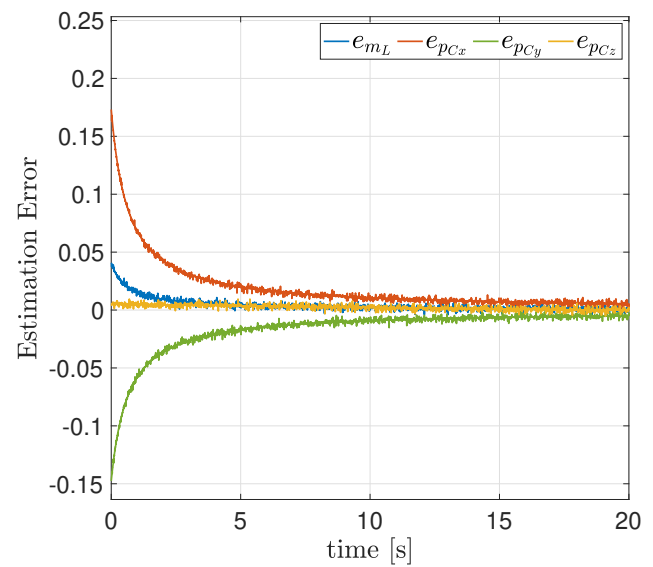

Fig. 4: The estimation errors (in kilograms for the mass and meters for the position coordinates) computed during the online estimation procedure. At time $t=0$ the errors correspond to the one obtained after the Initialization procedure.

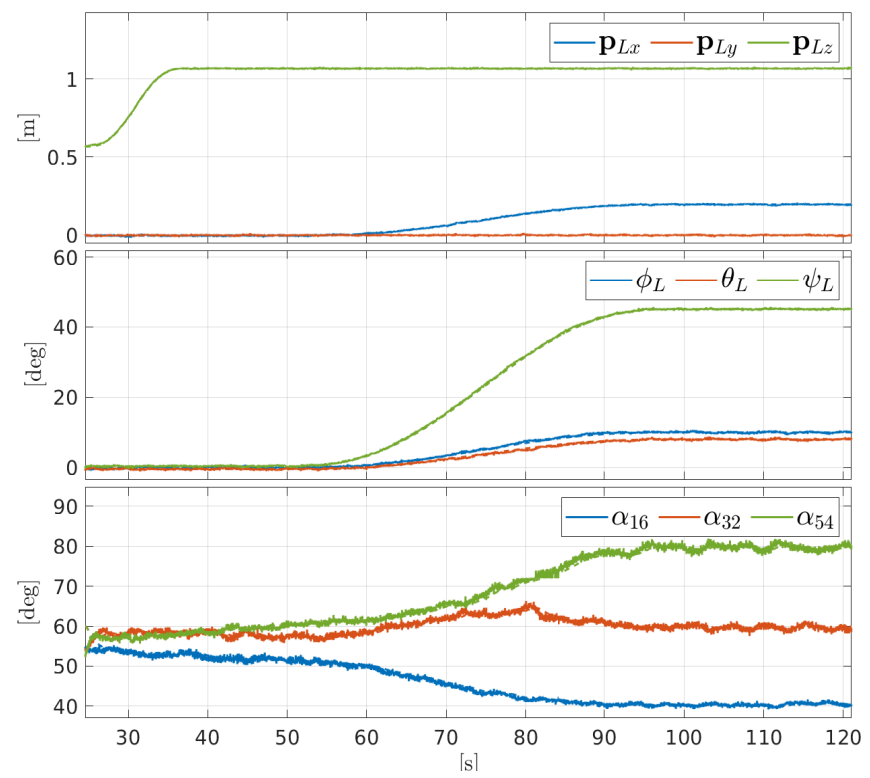

Fig. 5: The evolution of the configuration $\boldsymbol{q}=\left[\boldsymbol{p}_{L}^{\top} \boldsymbol{\eta}^{\top} \boldsymbol{\alpha}^{\top}\right]^{\top}$ of the Fly-Crane while tracking a desired trajectory and optimizing $\boldsymbol{\alpha}$.

procedure, described in Algorithm 1, with $\varsigma=15^{\circ}$. The outcome of this preliminary phase is an initial estimate $\widehat{\boldsymbol{p}}_{C}(0)=\left[\begin{array}{lll}-0.143-0.081 & 0.015\end{array}\right][\mathrm{m}]$ and $\widehat{m}=0.405[\mathrm{Kg}]$. The accuracy of this initial estimate is related to the noise provided by the simulation framework. To average out the noise, we run also the online estimation phase that leads the estimation errors to converge to zero, in average. The results of the online estimator are reported in Fig. 4. As can be seen, the estimation errors tend to go to zero thanks to the least-squares method, which is robust with respect to the noise thanks to its ability to fit all the data up to the current time. The online phase of the estimation procedure run at a frequency of $500[\mathrm{~Hz}]$.

\section{Validation of the Optimization Algorithm}

Once the estimation phase is completed, the system is demanded to track a trajectory which takes the system from its initial configuration, $\boldsymbol{q}^{d}(0)$ where $\boldsymbol{\alpha}=\left[\begin{array}{lll}60 & 60 & 60\end{array}\right]$ [deg], to a configuration $\boldsymbol{q}^{d}\left(T_{1}\right)$ where $\boldsymbol{p}_{L}^{d}=\left[\begin{array}{lll}0 & 0 & 1.5\end{array}\right][\mathrm{m}]$ and $\boldsymbol{\eta}^{d}=\left[\begin{array}{lll}0 & 0 & 0\end{array}\right][\mathrm{deg}]$ with $T_{1}=60[\mathrm{~s}]$, to the final desired configuration $\boldsymbol{q}^{d}\left(T_{2}\right)$ where $\boldsymbol{p}_{L}^{d}=\left[\begin{array}{lll}0.2 & 0 & 1.5\end{array}\right][\mathrm{m}], \boldsymbol{\eta}^{d}=$ [10 8 1 45$]$ [deg] with $T_{2}=120$ [s]. While the system tracks the trajectory, $\boldsymbol{\alpha}$ are optimized to distribute the entire payload among the robots in the best possible way. To better illustrate the performance of the algorithm, the optimization results will be compared with a not optimized case, where the desired angles of the configuration of the system are equal to $\boldsymbol{\alpha}_{c}=\left[\begin{array}{lll}38 & 56 & 46\end{array}\right][\mathrm{deg}]$. Notice that this particular choice leads to a feasible trajectory.

In Fig. 5, we present the results of the tracking of the desired trajectory while the optimization is running. The controller is considerably robust in maintaining the desired platform position and orientation while following the output of the optimization algorithm, i.e. $\boldsymbol{\alpha}_{\text {opt }}$, that varies significantly during the tracking task. On the other hand, Fig. 6a shows the optimized configuration compared to $\boldsymbol{\alpha}_{c}$. As can be seen, the optimization procedure forces the system to reach different angles $\boldsymbol{\alpha}$, if compared with the not optimized case, that reflects an optimal configuration with respect to the minimization of the total exerted actuation effort. Furthermore, Fig. $6 \mathrm{~b}$ shows how the cost function $\mu$ reaches a lower value as a result of the optimization process, when compared to the one obtained in the not optimized case. The optimization is running since the beginning of the task, in $\boldsymbol{q}^{d}(0)$, leading $\mu_{\text {opt }}$ to decrease and $\boldsymbol{\alpha}$ to move from their initial values. Then, at $T_{1}$, when the new robot arrangement $\boldsymbol{q}^{d}\left(T_{1}\right)$ is required, the evolution of the cost function $\mu_{o p t}$ is distinctly below the not optimized $\mu$, as a consequence of the $\alpha$ gradual variation. Then, the cost function keeps such beneficial gap until the end of the simulation. It is worth observing that the cost function rise is caused by the transition between two different system setups for which the required thrusts must be different enough among the three UAVs. For the sake of completeness, Fig. $6 c$ shows the trend of robot thrusts in the optimized case compared to not optimized case. As can be seen, the optimization algorithm reduces the dispersion of the three total thrusts by increasing $\boldsymbol{f}_{R 56_{o p t}}$ and decreasing of $\boldsymbol{f}_{R 12 \text { opt }}$ and $\boldsymbol{f}_{R 34_{o p t}}$. This causes the minimization of the maximum total thrust exerted by the system and, as a consequence, the extension of the overall flight time.

\section{CONCLUSIONS}

We have presented a novel parameters estimation strategy for towed-cable multi-robot systems and we showcased its performance on the so-called Fly-Crane system. Our analysis shows that the knowledge of the applied forces in two different static configurations is sufficient to estimate the mass and the center of mass of the loaded platform. We have shown how this estimation procedure can be useful to optimize the performance of the system in terms of energy. 


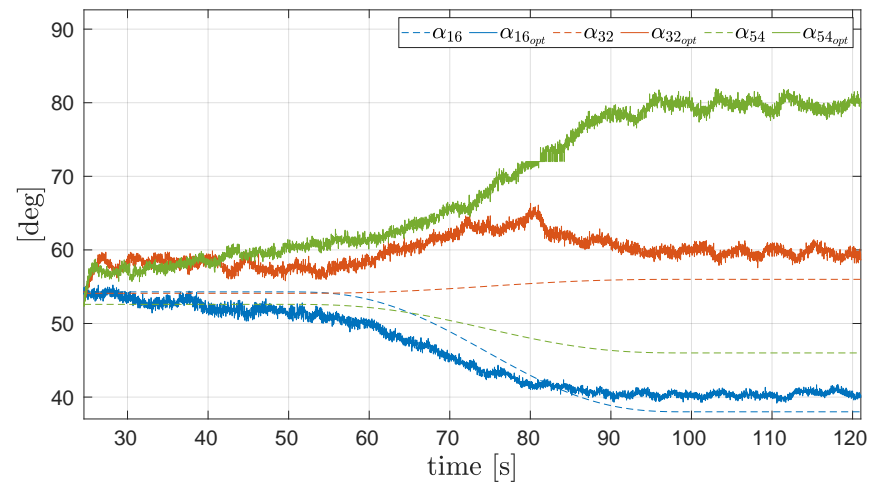

(a) The evolution of the angles $\boldsymbol{\alpha}$ in the case in which the optimization procedure is running (solid lines) and is not running (dashed lines).

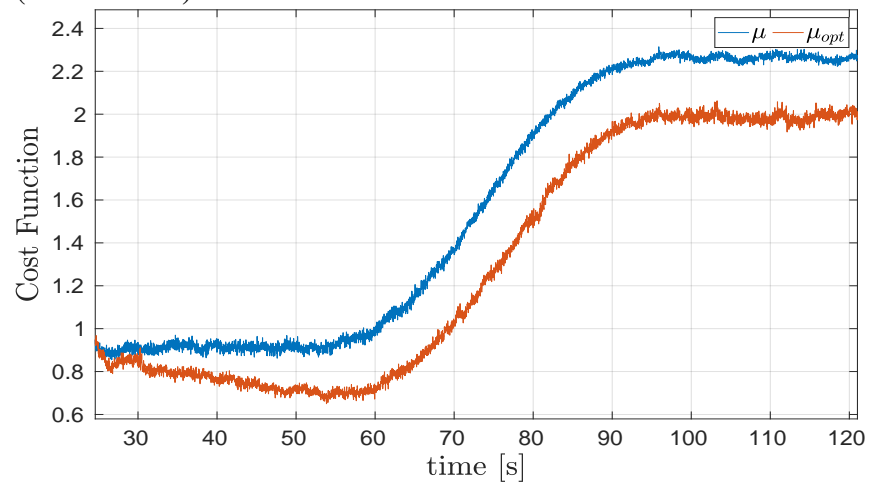

(b) The evolution of the cost function during the trajectory tracking: in red, the case with the optimization of $\boldsymbol{\alpha}$, i.e. $\boldsymbol{\alpha}=\boldsymbol{\alpha}_{\text {opt }}$, and, in blue, the case with constant $\boldsymbol{\alpha}=\boldsymbol{\alpha}_{c}$.

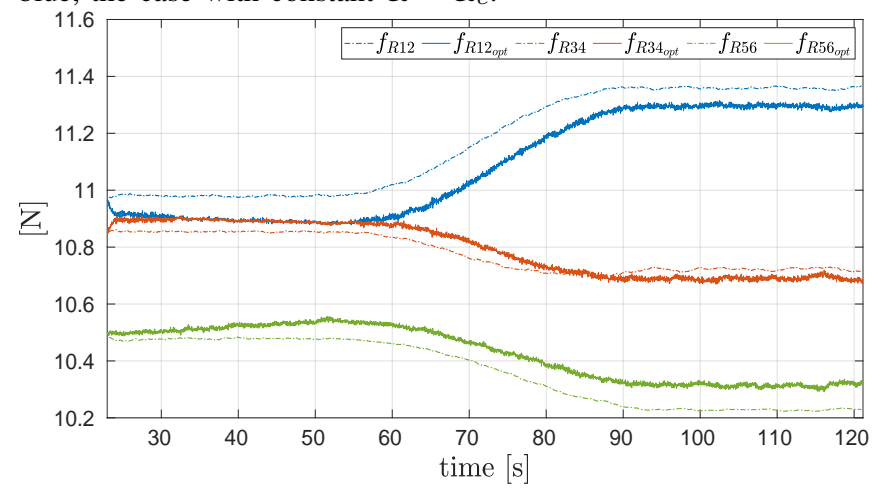

(c) The evolution of the UAV total thrusts in the optimized case (solid line) and not optimized case (dashed line).

Fig. 6: The comparison between the evolution of the Fly-Crane system in the not optimized case and optimized one

Finally, we have validated the proposed strategy by means of numerical simulations. Future work will deal with the analysis of the noise sensitivity of the estimation strategy and with the development of an online active sensing estimation procedure able to detect the best configurations given the noisy measurements.

\section{REFERENCES}

[1] H. G. d. Marina and E. Smeur, "Flexible collaborative transportation by a team of rotorcraft," in 2019 International Conference on Robotics and Automation (ICRA), May 2019, pp. 1074-1080.
[2] M. Bernard, K. Kondak, I. Maza, and A. Ollero, "Autonomous transportation and deployment with aerial robots for search and rescue missions," Journal of Field Robotics, vol. 28, no. 6, pp. 914-931, 2011.

[3] K. Sreenath and V. Kumar, "Dynamics, control and planning for cooperative manipulation of payloads suspended by cables from multiple quadrotor robots," in Robotics: Science and Systems, Berlin, Germany, June 2013.

[4] R. Connelly and S. Guest, "Frameworks, tensegrities and symmetry: Understanding stable structures," 2016.

[5] D. Prattichizzo and J. C. Trinkle, "Grasping," in Springer Handbook of Robotics, B. Siciliano and O. Khatib, Eds. Springer, 2008, pp. 671-700.

[6] C. Masone, H. H. Blthoff, and P. Stegagno, "Cooperative transportation of a payload using quadrotors: A reconfigurable cable-driven parallel robot," in 2016 IEEE/RSJ International Conference on Intelligent Robots and Systems (IROS), Oct 2016, pp. 1623-1630.

[7] M. Manubens, D. Devaurs, L. Ros, and J. Cortés, "Motion planning for 6-D manipulation with aerial towed-cable systems," in 2013 Robotics: Science and Systems, Berlin, Germany, May 2013.

[8] D. Six, S. Briot, A. Chriette, and P. Martinet, "The kinematics, dynamics and control of a flying parallel robot with three quadrotors," IEEE Robotics and Automation Letters, vol. 3, no. 1, pp. 559-566, Jan 2018.

[9] T. Lee, "Geometric control of quadrotor uavs transporting a cablesuspended rigid body," IEEE Transactions on Control Systems Technology, vol. 26, no. 1, pp. 255-264, 2017.

[10] M. Tognon, C. Gabellieri, L. Pallottino, and A. Franchi, "Aerial comanipulation with cables: The role of internal force for equilibria, stability, and passivity," IEEE Robotics and Automation Letters, Special Issue on Aerial Manipulation, vol. 3, no. 3, pp. 2577 - 2583, 2018.

[11] H. Lee, H. Kim, W. Kim, and H. J. Kim, "An integrated framework for cooperative aerial manipulators in unknown environments," IEEE Robotics and Automation Letters, vol. 3, no. 3, pp. 2307-2314, July 2018.

[12] D. Sanalitro, H. J. Savino, M. Tognon, J. Cortés, and A. Franchi, "Fullpose manipulation control of a cable-suspended load with multiple uavs under uncertainties," IEEE Robotics and Automation Letters, vol. 5, no. 2, pp. 2185-2191, 2020.

[13] D. Six, S. Briot, A. Chriette, and P. Martinet, "The kinematics, dynamics and control of a flying parallel robot with three quadrotors," IEEE Robotics and Automation Letters, vol. 3, no. 1, pp. 559-566, Jan 2018.

[14] N. Buaphim, K. Onsaard, P. Songoen, and T. Rungratgasame, "Some reviews on ranks of upper triangular block matrices over a skew field," International Mathematical Forum, vol. 13, no. 7, pp. 559-566, 2018

[15] H.Schwerdtfeger, Introduction to linear algebra and the theory of matrices, Noordhoff, Groningen, 1961.

[16] J. J. E. Slotine and W. Li, Applied nonlinear control. Prentice Hall, 1991

\section{APPENDIX}

Given the Gramian matrix defined as $\mathbf{G}=\widetilde{\mathbf{A}}^{T} \widetilde{\mathbf{A}}$, where $\widetilde{\mathbf{A}}=\left[\begin{array}{ll}\mathbf{A}_{1}^{T} & \mathbf{A}_{2}^{T}\end{array}\right]^{T}$ and

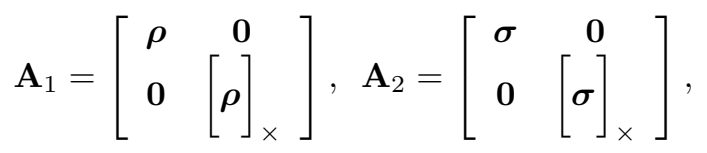

where $\boldsymbol{\rho}=\sum_{i=1}^{6}{ }^{P} \mathbf{f}_{i}\left(\overline{\boldsymbol{q}}_{1}\right), \boldsymbol{\sigma}=\sum_{i=1}^{6}{ }^{P} \mathbf{f}_{i}\left(\overline{\boldsymbol{q}}_{2}\right)$ and $\mathbf{f}_{i}(\boldsymbol{q})$ indicates the resulting force acting on the platform given the configuration $\boldsymbol{q}$.

Indicated with $\rho_{1,2,3}$ and $\sigma_{1,2,3}$ the components of, respectively, $\rho$ and $\sigma$, let us compute the eigenvalues of $\mathbf{G}$ by 
solving $\mathbf{G}-\lambda \mathbf{I}=0$ for $\lambda$ leading to the following values

$$
\begin{aligned}
\lambda_{1 / 2}= & \rho_{1}^{2}+\rho_{2}^{2}+\rho_{3}^{2}+\sigma_{1}^{2}+\sigma_{2}^{2}+\sigma_{3}^{2}=\boldsymbol{\rho}^{T} \boldsymbol{\rho}+\boldsymbol{\sigma}^{T} \boldsymbol{\sigma} \\
\lambda_{3}= & \frac{\rho_{1}^{2}+\rho_{2}^{2}+\rho_{3}^{2}+\sigma_{1}^{2}+\sigma_{2}^{2}+\sigma_{3}^{2}}{2}+ \\
& +\frac{1}{2}\left(\rho_{1}^{4}+2 \rho_{1}^{2} \rho_{2}^{2}+2 \rho_{1}^{2} \rho_{3}^{2}+2 \rho_{1}^{2} \sigma_{1}^{2}-2 \rho_{1}^{2} \sigma_{2}^{2}+\right. \\
& -2 \rho_{1}^{2} \sigma_{3}^{2}+8 \rho_{1} \rho_{2} \sigma_{1} \sigma_{2}+8 \rho_{1} \rho_{3} \sigma_{1} \sigma_{3}+\rho_{2}^{4}+ \\
& +2 \rho_{2}^{2} \rho_{3}^{2}-2 \rho_{2}^{2} \sigma_{1}^{2}+2 \rho_{2}^{2} \sigma_{2}^{2}-2 \rho_{2}^{2} \sigma_{3}^{2}+\rho_{3}^{4}+ \\
& +8 \rho_{2} \rho_{3} \sigma_{2} \sigma_{3}-2 \rho_{3}^{2} \sigma_{1}^{2}-2 \rho_{3}^{2} \sigma_{2}^{2}+2 \rho_{3}^{2} \sigma_{3}^{2}+ \\
& \left.+\sigma_{1}^{4}+2 \sigma_{1}^{2} \sigma_{2}^{2}+2 \sigma_{1}^{2} \sigma_{3}^{2}+\sigma_{2}^{4}+2 \sigma_{2}^{2} \sigma_{3}^{2}+\sigma_{3}^{4}\right)^{\frac{1}{2}}= \\
& =\frac{1}{2}\left(\boldsymbol{\rho}^{T} \boldsymbol{\rho}+\boldsymbol{\sigma}^{T} \boldsymbol{\sigma}\right)+\frac{1}{2} \xi^{\frac{1}{2}} \\
\lambda_{4}= & \frac{\rho_{1}^{2}+\rho_{2}^{2}+\rho_{3}^{2}+\sigma_{1}^{2}+\sigma_{2}^{2}+\sigma_{3}^{2}}{2}- \\
& +\frac{1}{2}\left(\rho_{1}^{4}+2 \rho_{1}^{2} \rho_{2}^{2}+2 \rho_{1}^{2} \rho_{3}^{2}+2 \rho_{1}^{2} \sigma_{1}^{2}-2 \rho_{1}^{2} \sigma_{2}^{2}+\right. \\
& -2 \rho_{1}^{2} \sigma_{3}^{2}+8 \rho_{1} \rho_{2} \sigma_{1} \sigma_{2}+8 \rho_{1} \rho_{3} \sigma_{1} \sigma_{3}+\rho_{2}^{4}+ \\
& +2 \rho_{2}^{2} \rho_{3}^{2}-2 \rho_{2}^{2} \sigma_{1}^{2}+2 \rho_{2}^{2} \sigma_{2}^{2}-2 \rho_{2}^{2} \sigma_{3}^{2}+\rho_{3}^{4}+ \\
& +8 \rho_{2} \rho_{3} \sigma_{2} \sigma_{3}-2 \rho_{3}^{2} \sigma_{1}^{2}-2 \rho_{3}^{2} \sigma_{2}^{2}+2 \rho_{3}^{2} \sigma_{3}^{2}+ \\
& \left.+\sigma_{1}^{4}+2 \sigma_{1}^{2} \sigma_{2}^{2}+2 \sigma_{1}^{2} \sigma_{3}^{2}+\sigma_{2}^{4}+2 \sigma_{2}^{2} \sigma_{3}^{2}+\sigma_{3}^{4}\right)^{\frac{1}{2}}= \\
& =\frac{1}{2}\left(\boldsymbol{\rho}^{T} \boldsymbol{\rho}+\boldsymbol{\sigma}^{T} \boldsymbol{\sigma}\right)-\frac{1}{2} \xi^{\frac{1}{2}}
\end{aligned}
$$

Moreover, let us simplify $\xi$ by writing the negative products as $-2 \rho_{i}^{2} \sigma_{j}^{2}=2 \rho_{i}^{2} \sigma_{j}^{2}-4 \rho_{i}^{2} \sigma_{j}^{2}$ and adding the terms $-4 \rho_{k}^{2} \sigma_{k}^{2}+$ $4 \rho_{k}^{2} \sigma_{k}^{2}$, for $k=1,2,3$, leading to

$$
\begin{aligned}
\xi= & \left(\rho_{1}^{4}+\rho_{2}^{4}+\rho_{3}^{4}+\sigma_{1}^{4}+\sigma_{2}^{4}+\sigma_{3}^{4}+2 \rho_{1}^{2} \rho_{2}^{2}+2 \rho_{1}^{2} \rho_{3}^{2}+\right. \\
& +2 \rho_{1}^{2} \sigma_{1}^{2}+2 \rho_{1}^{2} \sigma_{2}^{2}+2 \rho_{1}^{2} \sigma_{3}^{2}+2 \rho_{2}^{2} \rho_{3}^{2}+2 \rho_{2}^{2} \sigma_{1}^{2}+ \\
& +2 \rho_{2}^{2} \sigma_{2}^{2}+2 \rho_{2}^{2} \sigma_{3}^{2}+2 \rho_{3}^{2} \sigma_{1}^{2}+2 \rho_{3}^{2} \sigma_{2}^{2}+2 \rho_{3}^{2} \sigma_{3}^{2}+ \\
& \left.+2 \sigma_{1}^{2} \sigma_{2}^{2}+2 \sigma_{1}^{2} \sigma_{3}^{2}+2 \sigma_{2}^{2} \sigma_{3}^{2}\right)+ \\
& +\left(4 \rho_{1}^{2} \sigma_{1}^{2}+4 \rho_{2}^{2} \sigma_{2}^{2}+4 \rho_{2}^{2} \sigma_{2}^{2}+\right. \\
& \left.+8 \rho_{1} \rho_{2} \sigma_{1} \sigma_{2}+8 \rho_{2} \rho_{3} \sigma_{2} \sigma_{3}+8 \rho_{1} \rho_{3} \sigma_{1} \sigma_{3}\right)+ \\
& +\left(-4 \rho_{1}^{2} \sigma_{2}^{2}-4 \rho_{1}^{2} \sigma_{3}^{2}-4 \rho_{2}^{2} \sigma_{1}^{2}-4 \rho_{2}^{2} \sigma_{3}^{2}+\right. \\
& \left.-4 \rho_{3}^{2} \sigma_{1}^{2}-4 \rho_{3}^{2} \sigma_{2}^{2}-4 \rho_{1}^{2} \sigma_{1}^{2}-4 \rho_{2}^{2} \sigma_{2}^{2}-4 \rho_{2}^{2} \sigma_{2}^{2}\right) \\
= & \left(\boldsymbol{\rho}^{T} \boldsymbol{\rho}+\boldsymbol{\sigma}^{T} \boldsymbol{\sigma}\right)^{2}+4\left(\boldsymbol{\rho}^{T} \boldsymbol{\sigma}\right)^{2}-4 \boldsymbol{\rho}^{T} \boldsymbol{\rho} \boldsymbol{\sigma}^{T} \boldsymbol{\sigma} .
\end{aligned}
$$

